\title{
AVALIAÇÃO DO VOLUME DO DISCO ARTICULAR DA ATM POR MEIO DE IMAGENS DE RESSONÂNCIA MAGNÉTICA USANDO UM SOFTWARE DE ANÁLISE DE IMAGEM
}

\author{
TMJ ARTICULAR DISC VOLUME EVALUATION FROM MAGNETIC \\ RESONANCE IMAGING IN PATIENTS USING AN ANALYSIS SOFTWARE
}

\author{
Jaqueline Serra Martins* \\ Brunno M. Campos* \\ Ana Carla Raphaelli Nahás-Scocate ${ }^{* * *}$ \\ Acácio Fuziy** \\ Claudio Fróes de Freitas ${ }^{* * *}$ \\ Andre Luiz Ferreira Costa**
}

\section{RESUMO}

O objetivo deste estudo foi verificar a variação do volume do disco articular da ATM em pacientes com DTM e em indivíduos de controle, correlacionando-a com diagnóstico de imagem de RM e com dados clínicos. Foram analisados 29 exames de ressonância magnética entre pacientes e controles, totalizando 58 ATMs, em cortes sagitais sequenciais das imagens de RM. O software livre MRIcro foi utilizado para delineamento manual dos limites anatômicos do disco articular e obtenção o volume do disco. Para analisar a correlação entre os volumes dos discos e as variáveis clínicas e por imagem, empregou-se o teste de Kruskal-Wallis. Para caracterizar e comparar as variáveis entre gênero e idade foi aplicado o Teste t de Student independente. Observou-se uma correlação entre o deslocamento do disco ( $p$-valor $=0.001$ para ATM direita e $p$-valor $=0.002$ para ATM esquerda) e o volume do disco, que aumentou significativamente no grupo de pacientes. Não foi encontrada associação significativa entre gênero e volume dos discos direito e esquerdo (respectivamente; $p$-valor $=0,640$ e 0,390). Concluiu-se que o volume do disco articular aumentou com a presença de desarranjo intra-articular.

Descritores: Articulação temporomandibular • Processamento de imagem assistida por computador • Imagem por ressonância magnética.

\section{A BS TRACT}

The objective of this study was to measure the variation in the volume of temporomandibular joint disc in the patients with TMD compared with control individuals, in magnetic resonance images and clinical data. Twenty nine subjects have been analyzed between patients and control, totalizing $58 \mathrm{TMJ}$ 's in MR sequential sections. The software MRICro was used to anatomic delineation and to acquire the volume. Statistical analysis was conducted using Kruskal-Wallis test to correlate between disc volumes and clinical imaging variables. To compare the dependence on variables gender and age, Student Independence test was used. A correlation has been detected between disc displacement ( $p$-value $=0.001$ for right TMJ and $p$-value $=0.002$ for left TMJ) and disc volume, which exhibited significant increase in patients. No significant correlation has been observed between gender and left and right disc volumes (respectively; p-value =0,640 e 0,390). It was concluded that joint disc volume increased with the presence of internal derangement.

Descriptors: Temporomandibular joint • Image processing, computer-assisted $\bullet$ Magnetic resonance imaging.

\footnotetext{
* Discente do curso de Odontologia da Universidade Cidade de São Paulo - UNICID.

** Físico-médico do Laboratório de Neuroimagem da Faculdade de Ciências Médicas da Universidade Estadual de Campinas - UNICAMP.

*** Professor Associado do Programa de Mestrado Profissional em Ortodontia da Universidade Cidade de São Paulo - UNICID.
} 


\section{N T RO DUÇ ÃO}

A articulação temporomandibular (ATM) é uma das articulações mais complexas do corpo humano, sendo formada pela cabeça da mandíbula (côndilo) e a fossa mandibular do osso temporal, cartilagem articular e, interposto a elas, encontra-se o disco articular, que é de tecido fibroso denso, praticamente desprovido de vasos sanguíneos e fibras nervosas ${ }^{1,2,3,4}$, ${ }^{5}$. A morfologia normal do disco articular possui uma forma bicôncava (que é determinada pela morfologia da cabeça da mandíbula e da fossa mandibular), dando-Ihe um aspecto "gravata-borboleta" 6, 7, 8, 9 .

Há inúmeros trabalhos relatando as diferentes variações da morfologia articular e sua classificação ${ }^{10,11}$. As formas mais mencionadas e aceitas atualmente são: normal (bicôncava), biplanar, arredondada, biconvexas, hemiconvexa, dobrada e espessamento da banda posterior ${ }^{12,13,14}$.

A disfunção temporomandibular (DTM) é um distúrbio que envolve condições clínicas da ATM, músculos mastigatórios e estruturas anatômicas associadas ${ }^{4,}$ 15. A DTM envolve alterações anatômicas nas estruturas que compõem a ATM, sendo suscetível a alterações histopatológicas que podem culminar na degeneração dos tecidos componentes ${ }^{16}$. O deslocamento anterior do disco, com ou sem redução, é uma disfunção intracapsular que conduz a alterações degenerativas na superfície discal e planos ósseos articulares, assim como nos tecidos retrodiscais. Mais frequentemente, o disco é deslocado anteriormente ${ }^{8}$, produzindo alteração em sua anatomia $^{12}$.

Estudos histológicos mostraram que discos com anatomia anormal possuem alguns tipos componentes celulares de granulação e cicatrização, tais como: fibroblastos, fibrócitos, miofibroblastos ${ }^{17}$, enfatizando-se a modificação tecidual discal.

Há numerosos trabalhos na literatura que enfatizam a necessidade de se identificar os fatores envolvidos com a gênese e evolução da DTM ${ }^{18,19}$. Nenhum estudo evidenciou a participação do volume do disco articular.

Diante do exposto, surgiu o questiona- mento se, juntamente com o deslocamento do disco, haveria alteração da estrutura deste, com perda de volume e sua degradação. Visando responder a essa questão, realizamos o estudo a seguir.

\section{MÉTODOS}

Os procedimentos adotados nesta pesquisa estavam de acordo com as normas exigidas pela Comitê de Ética da Faculdade de Ciências Médicas da UNICAMP, onde esta pesquisa foi analisada e aprovada.

O material utilizado para a composição da amostra faz parte do acervo de imagens do Laboratório de Neuroimagem da Faculdade de Ciências Médicas da UNICAMP, composto de 29 imagens de ressonância magnética (RM) da ATM. As imagens foram adquiridas por meio de um aparelho de Ressonância Magnética Elcint Prestige (Haifa, Israel), operando em 2 Teslas, com bobinas bilaterais de três polegadas de diâmetro específicas para ATM, de acordo com os seguintes parâmetros: Imagens sagitais 'spin echo' (SE) ponderadas em T1 (TR $=650 \mathrm{msec}$, $\mathrm{TE}=22$ msec, matrix $=316 \times 240$, flip 160o, espessura de $1,5 \mathrm{~mm}$, field of view = 10x10, NEX 1) adquiridas em boca aberta e fechada. T2-weighted FSE sagital (TR = $5300 \mathrm{msec}, \mathrm{TE}=90 \mathrm{msec}$, matrix $=216 \mathrm{x}$ 216, flip 160o, espessura de 1,5mm, field of view $=12 \times 12$, NEX 2).

Selecionamos do banco de dados imagens no formato DICOM, as quais foram convertidas, pelo software MRIcro, em formato ANALYSE. Para segmentação do disco foi utilizado o Software MRIcro (http://www.sph.sc.edu/comd/rorden/), empregando os recursos do mesmo para melhor visualização do disco. Para se obter o volume do disco articular foi utilizada a chamada segmentação de imagens. Segmentação é um processo que objetiva separar as regiões que compõem uma imagem, abrangendo todos os voxels dentro da mesma faixa de treshold demarcada $^{4}$, proporcionando o volume total da estrutura segmentada.

A segmentação manual foi executada em sequências T1, desenhando-se uma linha contornando o disco e incluindo-o
MARTINS JS

CAMPOS BM

NAHÁS-SCOCATE ACR

FUZIY A

FREITAS CF

COSTA ALF

AVALIAÇÃO DO

VolUME dO DISCO

ARTICULAR DA

ATM POR MEIO

DE IMAGENS DE

RESSONÂNCIA

MAGNÉTICA USANDO

UM SOFTWARE DE

ANÁLISE DE IMAGEM

\section{9}

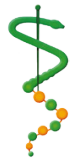

REV. ODONTOL.

UNIV, CID, SÃO

PAULO

2015; 27(2): 118 -

25, MAIO-AGO 
CAMPOS BM

NAHÁS-SCOCATE ACR

FUZIY A

FREITAS CF

COSTA ALF

AVALIAÇÃO DO VOLUME DO DISCO

ARTICULAR DA ATM POR MEIO DE IMAGENS

DE RESSONÂNCIA MAGNÉTICA USANDO UM SOFTWARE DE ANÁLISE DE IMAGEM
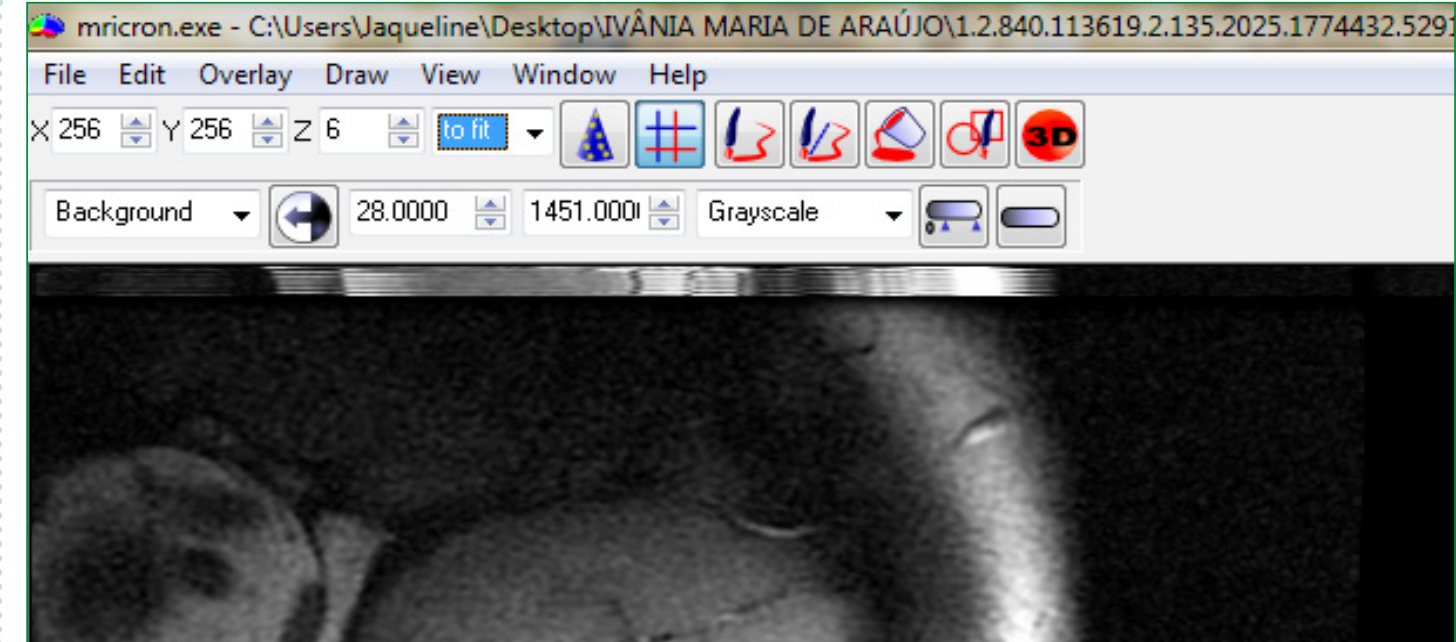


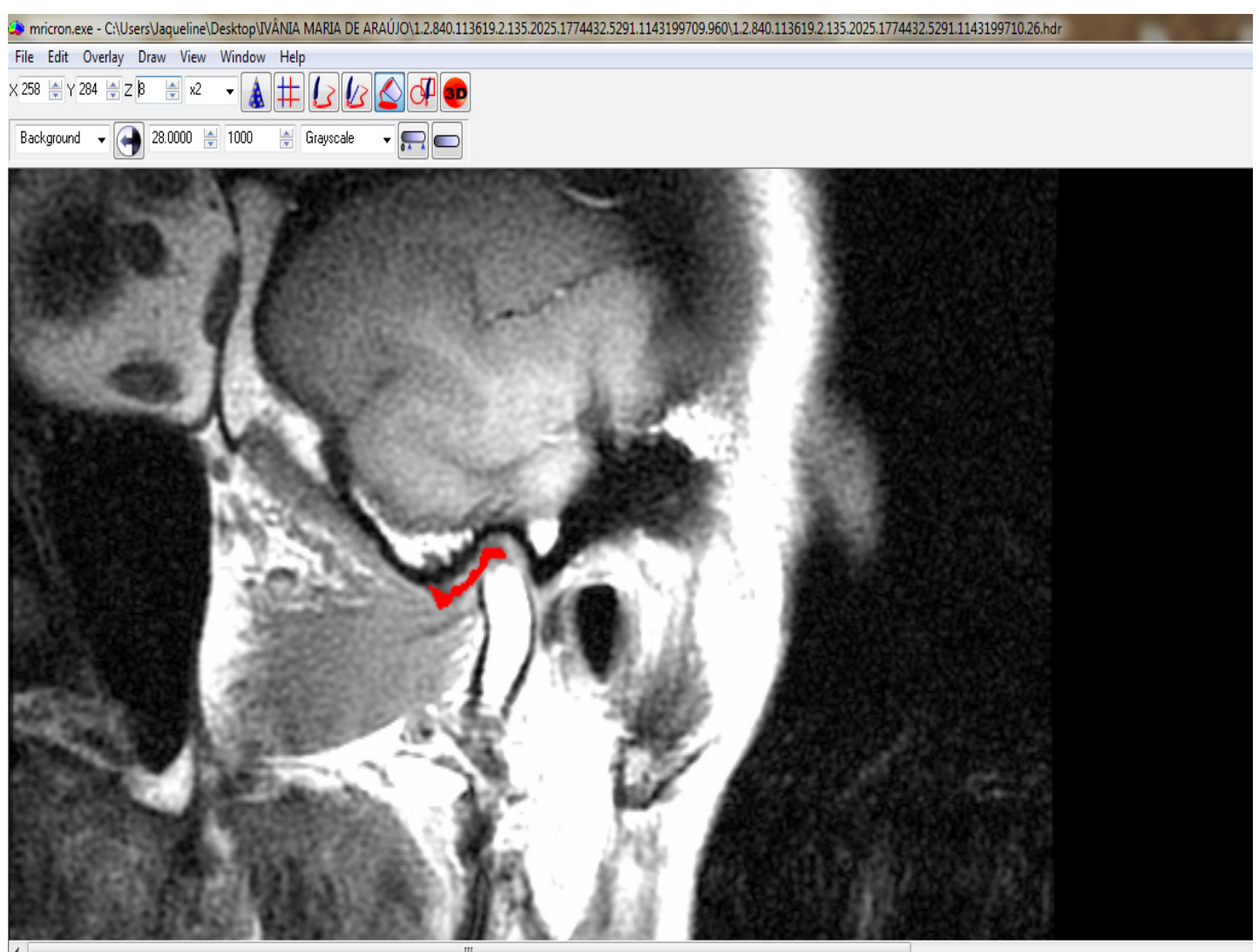

$1 \times 8 \times 53=46.00000$

Figura 2: Disco segmentado e preparado para a extração do volume.

rando-se as variáveis entre sexos e faixas etárias, foi aplicado o Teste t de Student independente. Adotou-se o nível de significância de 5\%, ou seja, $\mathrm{p}<0.05$.

\section{RESULTADOS}

De acordo com nosso trabalho, a idade dos pacientes selecionados variou de 17 a 71 anos (Tabela 1). As imagens foram avaliadas por um único examinador antecipadamente calibrado, com treinamento em DTM. Os parâmetros considerados pelo examinador foram: posição e redução do disco articular. As avaliações clínicas foram feitas em uma pesquisa anterior pelo mesmo examinador. O diagnóstico de dor miofascial baseou-se na presença de dor autorreferida ao repouso e sensibilidade à palpação de três ou mais músculos em locais definidos. A abertura da boca foi avaliada pela mensuração da distância entre o incisivo inferior e superior por meio de paquímetro digital. Avaliou-se, ainda, a presença de desvio click articular e dor nos movimentos mandibulares. As análises clínicas permitiram caracterizar os indivíduos com DTM (pacientes) e sem
DTM (controles). Os dados gerais podem ser observados na Tabela 1.

Não foi encontrada associação significativa entre gênero e volume dos discos direito e esquerdo (respectivamente; p-valor $=0,640$ e 0,390$)$. Houve associação entre o deslocamento do disco ( $\mathrm{N}$ : normal; DDR: deslocamento de disco com redução; DDSR: deslocamento de disco sem redução), tanto para o direito ( $p$-valor $=0.001$ ), quanto para o esquerdo ( $p$-valor $=0.002$ ). O volume do disco aumentou significantemente nas ATMs com deslocamento de disco (Figuras 3 e 4).

Houve correlação entre DTM e o volume do disco articular direito ( $p$-valor $=0,016)$, com o aumento do volume do disco quando os indivíduos apresentavam DTM. Não foi encontrada associação entre presença de DTM e volume do disco esquerdo (p-valor $=0,085)$ (Figuras 5 e 6).

\section{I SCUSSÃO}

Por meio deste estudo, pudemos obserça de deslocamento de disco e o volume do disco articular ( $p=0.001$, lado direito; var que houve diferença quanto à presen-
MARTINS JS

CAMPOS BM

NAHÁS-SCOCATE ACR

FUZIY A

FREITAS CF

COSTA ALF

AVALIAÇÃO DO

VOLUME DO DISCO

ARTICULAR DA

ATM POR MEIO

DE IMAGENS DE

RESSONANNCIA

MAGNÉTICA USANDO

UM SOFTWARE DE

ANÁLISE DE IMAGEM

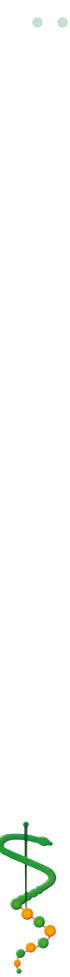

REV. ODONTOL.

UnIV. CID, São

PAULO

2015; 27(2): 118 -

25, MAIO-AGO 


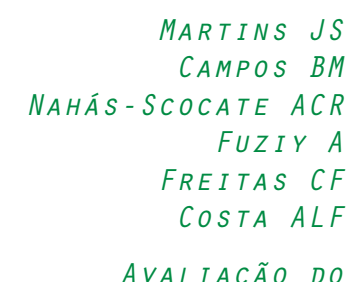

VOLUME DO DISCO

ARTICULAR DA ATM POR MEIO DE IMAGENS

DE RESSONÂNCIA MAGNÉTICA USANDO UM SOFTWARE DE ANÁLISE DE IMAGEM

REV, ODONTOL

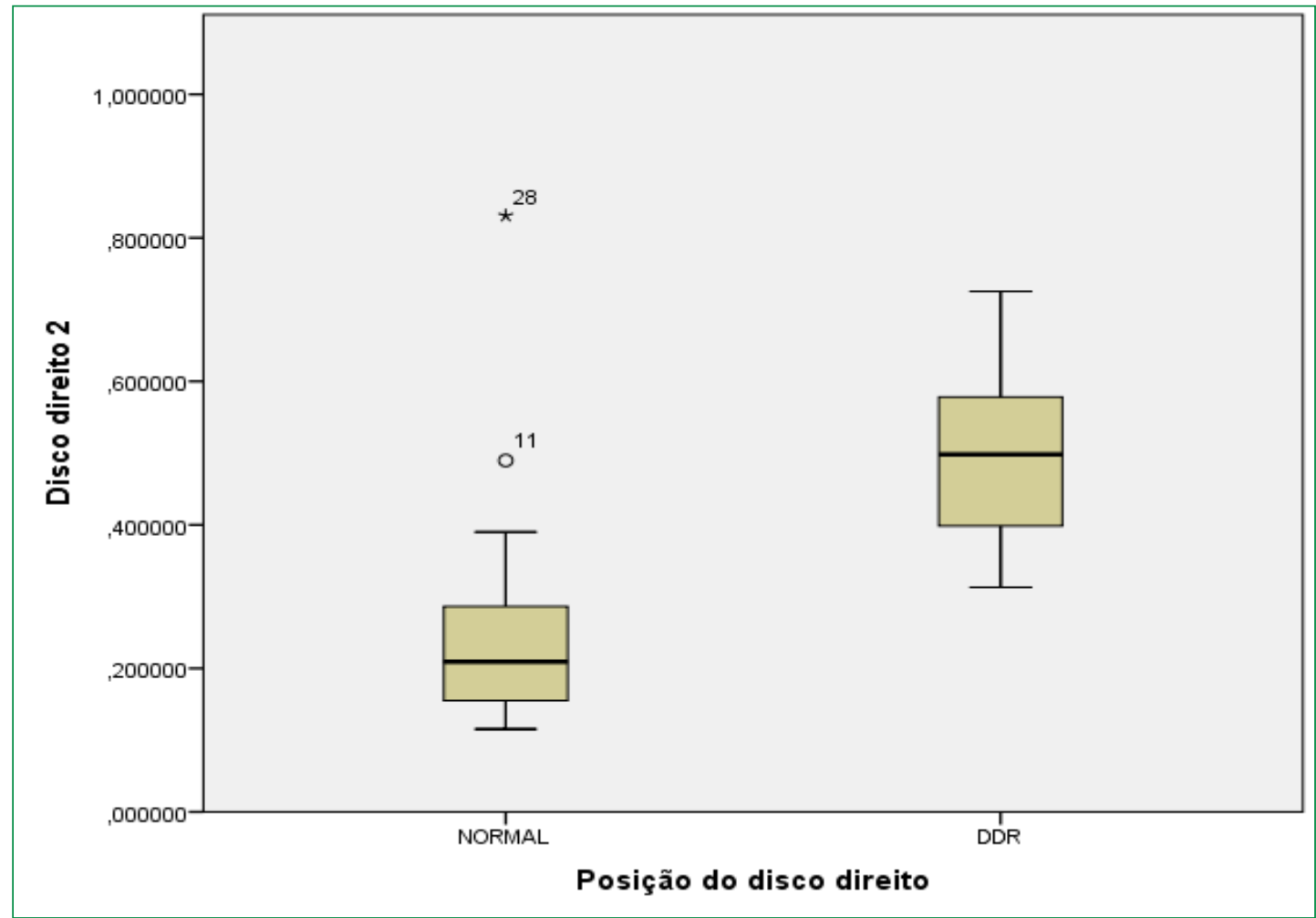

Figura 3: Deslocamento do disco articular e volume (lado direito)

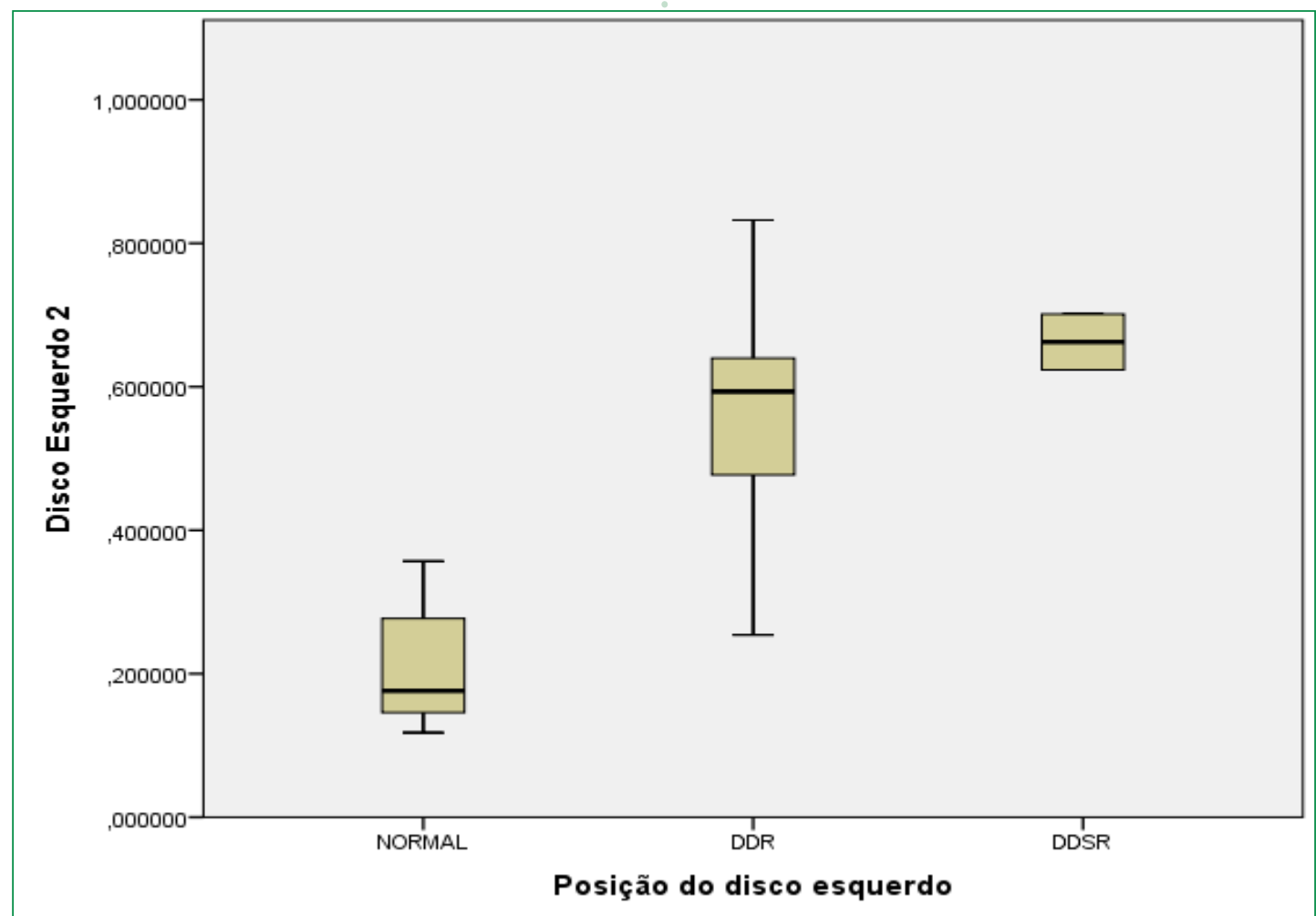

Figura 4: Deslocamento do disco articular e volume (lado esquerdo)

$\mathrm{p}=0.002$, lado esquerdo); notou-se que o de disco do que em indivíduos saudáveis. volume do disco articular aumentou com A oclusão dentária apresenta papel impora presença de desarranjo intra-articular. tante como fator predisponente que alteKurita et al. ${ }^{13}$ (1989) e Wang et al..$^{17}$ (2009) relataram que os discos da ATM são mais espessos em pacientes com deslocamento ra o sistema mastigatório suficientemente para incrementar o risco de desenvolver disfunção de $\mathrm{ATM}^{7}$. Os hábitos parafun- 


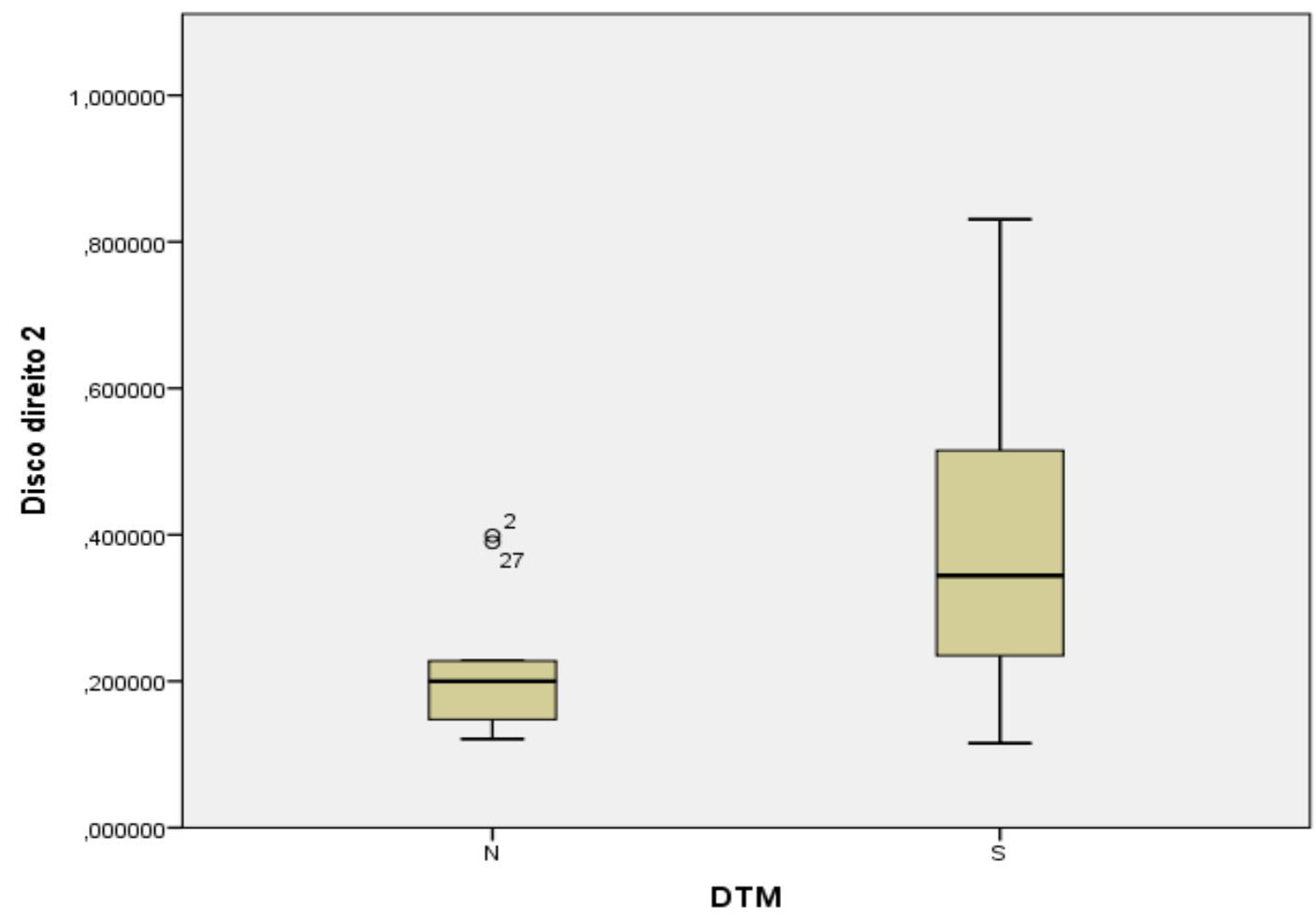

MARTINS JS

CAMPOS BM

NAHÁS-SCOCATE ACR

FUZIY A

FREITAS CF

COSTA ALF

AVALIAÇÃO DO

VOLUME DO DISCO

ARTICULAR DA

ATM POR MEIO

DE IMAGENS DE

RESSONÂNCIA

MAGNÉTICA USANDO

UM SOFTWARE DE

ANÁLISE DE IMAGEM

Figura 5: Relação entre presença de DTM e volume do disco direito

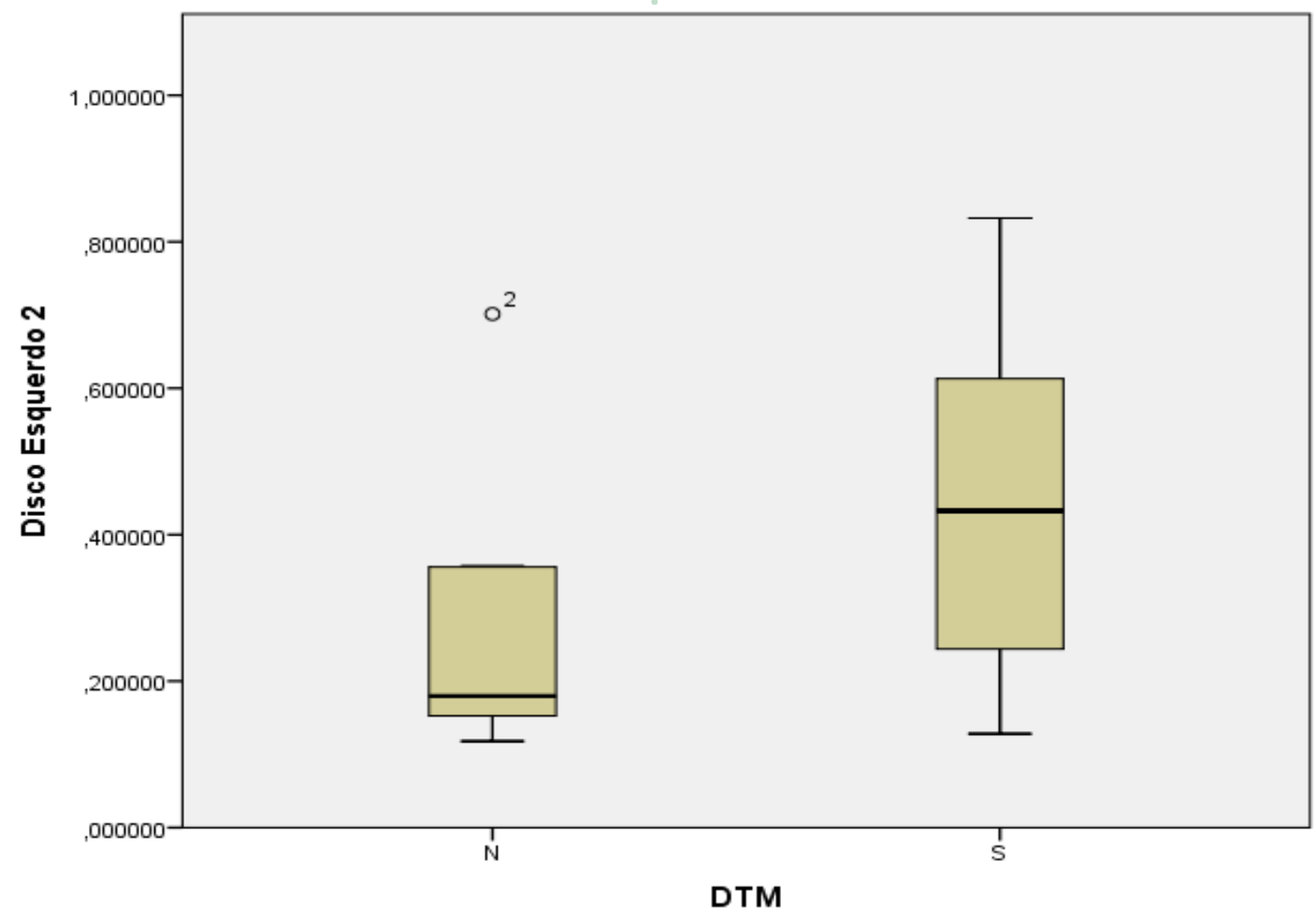

Figura 6: Relação entre presença de DTM e volume do disco direito e esquerdo

cionais e a má-oclusão dentária induzem a produzir micro-traumas na ATM desenvolvendo-se, assim, lesões degenerativas no côndilo e no disco articular, e este tende a se espessar para compensar esse dano ${ }^{13}$.
Em nosso estudo, avaliamos a presença de DTM e o volume articular. Encontramos relação com o aumento do volume do disco do lado direito $(p=0,016)$, entretanto, do lado esquerdo não houve correlação $(p=0,085)$. Estudos mostram que o

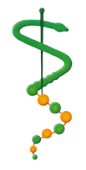

REV. ODONTOL.

UNIV, C I D, SÃO

PAUlo

2015; 27(2): 118 -

25, MAIO-AGO 
MARTINS JS

CAMPOS BM

NAHÁS-SCOCATE ACR

FUZIY A

FREITAS CF

COSTA ALF

AVALIAÇÃO DO

VOLUME DO DISCO

ARTICULAR DA ATM POR MEIO DE IMAGENS

DE RESSONÂNCIA MAGNÉTICA USANDO UM SOFTWARE DE ANÁLISE

DE IMAGEM

\section{4}

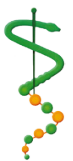

REV, ODONTOL,

UNIV. CID, SÃO

PAULO

$2015 ; 27(2): 118$ -

25, MAIO-AGO ato de ranger os dentes gera uma sobrecarga nos músculos da mastigação que pode facilitar o desenvolvimento da DTM em indivíduos. É interessante observar que os indivíduos muitas vezes tendem a apresentar tendência de mastigação unilateral, o que pode explicar nossos resultados.

Amaral et al. ${ }^{6}$, em 2013, afirmaram que as alterações morfológicas no disco são influenciadas pelo tipo de deslocamento. Em nossos resultados, comparando o volume do disco quando ocorria seu deslocamento, notamos um maior espessamento quando o disco não é recapturado, indicando desenvolvimento de alterações celulares.

Kurita et al. ${ }^{13}$, em 1989, afirmaram que a espessura dos discos com deformidades retirados cirurgicamente era maior do que a dos discos normais. Assim, uma camada superficial proliferativa, condrócitos com formação de cartilagem e aumento de fibroblastos e vasos foram

\section{REFERÊNCIAS}

1. Tallents RH, Katzberg RW, Murphy W, Proskin H. Magnetic resonance imaging findings in asymptomatic volunteers and symptomatic patients with temporomandibular disorders. J Prosthet Dent 1996 May;75(5):529-33.

2. Thompson JR. Triad of dentistry: diagnosis and treatment. United States of America: Northwestern University Dental School; 1994.

3. Bumann A, Lotzmann U. Disfunção temporomandibular: diagnóstico funcional e princípios terapêuticos. In: Atlas colorido de odontologia. Porto Alegre: Artmed; 2002.

4. Costa AL, Yasuda CL, Appenzeller S, Lopes SL, Cendes F. Comparison of conventional MRI and 3D reconstruction model for evaluation of temporomandibular joint. Surg Radiol Anat 2008 Nov;30(8):663-7.

5. Heffez LB, Mafee $M$, Rosenberg $H$. Atlas of temporomandibular joint imaging. EUA: Lippincott Williams and Wilkins; 1995. mais comuns nas amostras cirúrgicas do que nas articulações normais, que foram encontradas nos cortes histológicos, sugerindo que nas articulações que apresentam algum tipo de DTM ocorre um espessamento do disco como forma de autoproteção, e sugerindo que a deformação é resultado da proliferação tecidual como mecanismo de proteção.

\section{CONCLUSÃO}

Por meio deste estudo, pudemos observar que:

Houve diferença quanto à presença de deslocamento de disco e o volume do disco articular lado direito e lado esquerdo.

Notou-se que o volume do disco articular aumentou com a presença de desarranjo intra-articular, principalmente com deslocamento de disco sem redução.

AGRADECIMENTOS à FAPESP pela concessão da bolsa de iniciação científica (Processo FAPESP 2013/09659-5).
6. Amaral R O, Damasceno NN, de Souza LA, Devito KL. Magnetic resonance images of patients with temporomandibular disorders: prevalence and correlation between disk morphology and displacement. Eur J Radiol 2013 Jun;82(6):990-4.

7. Costa AL, D'Abreu A, Cendes F. Temporomandibular joint internal derangement: association with headache, joint effusion, bruxism, and joint pain. J Contemp Dent Pract 2008 9(6):9-16.

8. Taskaya-Yilmaz N, Ogutcen-Toller M. Magnetic resonance imaging evaluation of temporomandibular joint disc deformities in relation to type of disc displacement. J Oral Maxillofac Surg 2001 Aug;59(8):860-5; discussion 5-6.

9. Porto VC. Avaliação da posição do disco articular em pacientes usuários de dentaduras duplas e portadores de sons articulares por meio de ressonância magnética da ATM [Tese]. Bauru, SP: Faculdade de Odontologia de Bauru; 2002. 
10. Milano V, Desiate A, Bellino R, Garofalo T. Magnetic resonance imaging of temporomandibular disorders: classification, prevalence and interpretation of disc displacement and deformation. Dentomaxillofac Radiol 2000 Nov;29(6):352-61.

11. Katzberg RW, Tallents RH. Normal and abnormal temporomandibular joint disc and posterior attachment as depicted by magnetic resonance imaging in symptomatic and asymptomatic subjects. J Oral Maxillofac Surg 2005 Aug;63(8):1155-61.

12. Incesu L, Taskaya-Yilmaz N, Ogutcen-Toller M, Uzun E. Relationship of condylar position to disc position and morphology. Eur J Radiol 2004 Sep;51(3):269-73.

13. Kurita K, Westesson PL, Sternby $\mathrm{NH}$, Eriksson L, Carlsson LE, Lundh $\mathrm{H}$, et al. Histologic features of the temporomandibular joint disk and posterior disk attachment: comparison of symptom-free persons with normally positioned disks and patients with internal derangement. Oral Surg Oral Med Oral Pathol 1989 Jun;67(6):635-43.

14. Orhan K, Nishiyama H, Tadashi S, Murakami S, Furukawa S. Comparison of altered signal intensity, position, and morphology of the TMJ disc in MR images corrected for variations in surface coil sensitivity. Oral Surg Oral Med Oral Pathol Oral Radiol Endod 2006 Apr;101(4):515-22.
15. Tomas X, Pomes J, Berenguer J, Quinto L, Nicolau C, Mercader JM, et al. MR imaging of temporomandibular joint dysfunction: a pictorial review. RadioGraphics 2006 26(3):765-81.

16. Matsumoto T, Inayama M, Tojyo I, Kiga N, Fujita S. Expression of hyaluronan synthase 3 in deformed human temporomandibular joint discs: in vivo and in vitro studies. Eur / Histochem 2010 54(4):e50.

17. Wang M, Cao H, Ge Y, Widmalm SE. Magnetic resonance imaging on TMJ disc thickness in TMD patients: a pilot study. J Prosthet Dent 2009 Aug;102(2):89-93.

18. Moen K, Hellem S, Geitung JT, Skartveit L. A practical approach to interpretation of MRI of the temporomandibular joint. Acta Radiol 2010 Nov;51(9):1021-7.

19. Larheim TA. Role of magnetic resonance imaging in the clinical diagnosis of the temporomandibular joint. Cells Tissues Organs 2005 180(1):6-21.

Recebido em 16/12/2015

Aceito em 16/12/2015
MARTINS JS

CAMPOS BM

NAHÁS-SCOCATE ACR

FUZIY A

FREITAS CF

COSTA ALF

AVALIAÇÃO DO

VOLUME DO DISCO

ARTICULAR DA

atM POR MEIO

DE IMAGENS DE

RESSONÂNCIA

MAGNÉTICA USANDO

UM SOFTWARE DE

ANÁLISE DE IMAGEM

\section{5}

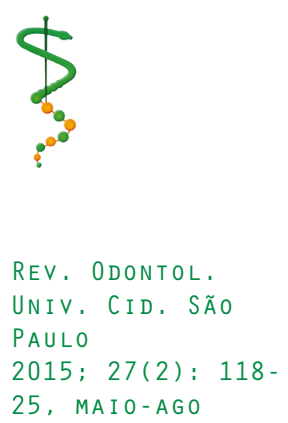

